Evidence and relevance of spatially chaotic magnetic field lines in MCF devices

This content has been downloaded from IOPscience. Please scroll down to see the full text.

2017 Plasma Phys. Control. Fusion 59034005

(http://iopscience.iop.org/0741-3335/59/3/034005)

View the table of contents for this issue, or go to the journal homepage for more

Download details:

IP Address: 200.0.233.51

This content was downloaded on 13/03/2017 at 12:42

Please note that terms and conditions apply.

You may also be interested in:

Resonant magnetic perturbations of edge-plasmas in toroidal confinement devices

TE Evans

Energetic particle physics in fusion research in preparation for burning plasma experiments

N.N. Gorelenkov, S.D. Pinches and K. Toi

Physics of collapse events in toroidal plasmas

S-I Itoh, K Itoh, H Zushi et al.

Local properties of magnetic reconnection in nonlinear resistive- and extended-magnetohydrodynamic toroidal simulations of the sawtooth crash

M T Beidler, P A Cassak, S C Jardin et al.

Non-linear phenomena in tokamak plasmas

B B Kadomtsev

Sawtooth mitigation in 3D MHD tokamak modelling with applied magnetic perturbations

D Bonfiglio, M Veranda, S Cappello et al.

Macroscopic stability of high MAST plasmas

I.T. Chapman, W.A. Cooper, J.P. Graves et al.

Dynamics of heavy impurities in non-linear MHD simulations of sawtoothing tokamak plasmas Jae-H Ahn, X Garbet, H Lütjens et al. 


\title{
Evidence and relevance of spatially chaotic magnetic field lines in MCF devices
}

\author{
M-C Firpo ${ }^{1}$, A F Lifschitz ${ }^{2}$, W Ettoumi ${ }^{1,3}$, R Farengo ${ }^{4}$, H E Ferrari ${ }^{4,5}$ and \\ P L García-Martínez ${ }^{5}$ \\ ${ }^{1}$ Laboratoire de Physique des Plasmas, CNRS—Ecole Polytechnique, F-91128 Palaiseau cedex, France \\ ${ }^{2}$ Laboratoire d'Optique Appliquée, ENSTA, CNRS, Ecole Polytechnique, F-91761 Palaiseau, France \\ ${ }^{3}$ Université de Genève, GAP-Biophotonics, Chemin de Pinchat 22, 1211 Geneva 4, Switzerland \\ ${ }^{4}$ Centro Atómico Bariloche (CNEA) and Instituto Balseiro (UNC-CNEA), San Carlos de Bariloche, RN \\ 8400, Argentina \\ ${ }^{5}$ Consejo Nacional de Investigaciones Científicas y Técnicas (CONICET), Bariloche, Argentina \\ E-mail: marie-christine.firpo@lpp.polytechnique.fr
}

Received 29 September 2016, revised 8 December 2016

Accepted for publication 5 January 2017

Published 31 January 2017

\begin{abstract}
Numerical evidence for the existence of spatially chaotic magnetic field lines about the collapse phase of tokamak sawteeth with incomplete reconnection is presented. This uses the results of extensive test particle simulations in different sets of electromagnetic perturbations tested against experimental JET measurements. In tokamak sawteeth, that form a laboratory prototype of magnetic reconnection, the relative magnetic perturbation $\delta B / B$ may reach a few percents. This does not apply to tokamak operating regimes dominated by turbulence where $\delta B / B$ is usually not larger than $10^{-4}$. However, this small magnetic perturbation being sustained by a large spectrum of modes is shown to be sufficient to ensure the existence of stochastic magnetic field lines. This has important consequences for magnetic confinement fusion where electrons are dominantly governed by the magnetic force. Indeed some overlap between magnetic resonances can locally induce chaotic magnetic field lines enabling the spatial redistribution of the electron population and of its thermal content. As they are the swiftest plasma particles, electrons feed back the most rapid perturbations of the magnetic field.
\end{abstract}

Keywords: magnetic confinement fusion, chaotic magnetic field lines, stochasticity, magnetic turbulence

(Some figures may appear in colour only in the online journal)

\section{Introduction}

Historically the modeling of transport processes in tokamaks relied first on a classical transport picture, where thermalization is mediated by particle-to-particle collisions. Yet the first estimates for the heat losses derived from this classical approach happened to be largely insufficient to account for experimental measurements. Later, Galeev and Sagdeev [1] revised the classical theory by taking into account the toroidal effects inducing the existence of orbits having a width much larger than the Larmor radius, the so-called banana orbits: their theory became known as the neoclassical theory. Even if the cross-field transport is substantially augmented compared with the classical theory, neoclassical estimates remain typically about one to two orders of magnitude smaller than actual tokamak losses. The reason behind this failure is the low collisionality of tokamak plasmas. Indeed because collisionality decreases strongly with plasma temperature, as $T_{e}^{-3 / 2}$ [2], diluted hot plasmas are intrinsically low collisional so that the physics of hot plasmas in magnetic confinement devices for fusion applications is dominated by collective effects.

The picture that emerges is that the paths to transport and thermalization in magnetic confinement fusion (MCF) devices may be separated in two distinct categories [3]. There is first an extrinsic path, that is non-cancelable, being due to the granularity of matter, that involves the particle-particle collisions. And there is a second, dominant, path that is intrinsic, 
collective and collisionless. Both paths should ultimately be reduced and controlled to optimize fusion performance $[4,5]$.

Neglecting collisions, the relevant mathematical frame to describe MCF plasma physics is the Vlasov equation for the distribution functions, $f_{s}(\mathbf{v}, \mathbf{r}, t)$, of the various particle species

$$
\frac{\partial f_{s}}{\partial t}+\mathbf{v} \cdot \frac{\partial f_{s}}{\partial \mathbf{r}}+\frac{q_{s}}{m_{s}}(\mathbf{E}+\mathbf{v} \times \mathbf{B}) \cdot \frac{\partial f_{s}}{\partial \mathbf{v}}=0,
$$

where the electric and magnetic fields, $\mathbf{E}$ and $\mathbf{B}$, are the sums of external and self-consistent fields and where the time $t \in[0 ; T]$. Let us define $f_{s 0}(\mathbf{v}, \mathbf{r})=f_{s}(\mathbf{v}, \mathbf{r}, t=0)$.

Solving the Vlasov equation (1) amounts to find the characteristics $\mathbf{R}$ and $\mathbf{V}$ that solve the system of differential equations for $0<\tau<T$

$$
\begin{gathered}
\frac{\mathrm{d} \mathbf{R}}{\mathrm{d} \tau}=\mathbf{V}(\tau), \\
\frac{\mathrm{d} \mathbf{V}}{\mathrm{d} \tau}=\frac{q_{s}}{m_{s}}\{\mathbf{E}[\mathbf{R}(\tau), \tau]+\mathbf{V}(\tau) \times \mathbf{B}[\mathbf{R}(\tau), \tau]\},
\end{gathered}
$$

with the conditions $\mathbf{R}(\tau=t)=\mathbf{r}$ and $\mathbf{V}(\tau=t)=\mathbf{v}$. Then the solution to the Vlasov equation at time $t$ is

$$
f_{s}(\mathbf{v}, \mathbf{r}, t)=f_{s 0}(\mathbf{V}(0), \mathbf{R}(0)) .
$$

What can be gained from this simple mathematical consideration? Neglecting collisions means that all MCF plasma particles interact only with the electromagnetic fields according to equations (2)-(3). This may be viewed as a validation for test-particle approaches in MCF plasmas. Indeed, if one provisionally discards self-consistency and approximates the electromagnetic fields with reasonably physical models, then doing statistics on test particles amounts to solve the (non self-consistent) Vlasov equation. Considering just equations (2)-(3) means a drastic reduction of the problem dimension and a simplification of the dynamics integration. Although the solution is not exact, being non self-consistent, one can use test-particle approaches to test different sets of electromagnetic field evolutions and approach the realistic one by confrontation to experimental measurements. This is indeed the route that will be presented in section 2 to test indirectly the regular or chaotic nature of magnetic field lines during some JET sawtooth collapses.

The principle of MCF is that plasma confinement is ensured by a strong, static, magnetic field $\mathbf{B}_{0}$, so that at the dominant order the dynamics of charged particles obey

$$
m \frac{\mathrm{d} \mathbf{v}}{\mathrm{d} t}=q \mathbf{v} \times \mathbf{B}_{0} .
$$

This equation ensures that the charged particle is confined, spiraling along $\mathbf{B}_{0}$, and that its energy remains constant. This conservative property is favorable for plasma insulation. Yet the initial plasma formation and its subsequent heating need to be driven by electric fields, which can be inductive as in tokamaks or high-frequency as in stellarators. The full, general, particle equation of motion is eventually of the type

$$
m \frac{\mathrm{d} \mathbf{v}}{\mathrm{d} t}=q \mathbf{v} \times \mathbf{B}_{0}+q \mathbf{E}+q \mathbf{v} \times \delta \mathbf{B},
$$

where the electric, $\mathbf{E}$, and magnetic, $\mathbf{B}_{0}+\boldsymbol{\delta} \mathbf{B}$, fields are related by Faraday's law.

Let us estimate the strength of the electric force on charged particles. From Faraday's law, we have $E_{\mathbf{k}} \sim \omega_{\mathbf{k}} \delta B_{\mathbf{k}} / k$. In situations of turbulence, typical fluctuation frequencies $\omega_{\mathbf{k}}$ have for instance been reported to be in the range $10^{4}-10^{5} \mathrm{~Hz}$ in tokamaks for centimeter wavelengths [6]. Consequently, as far as electrons are concerned, having typical velocities of $4 \times 10^{7} \mathrm{~m} \mathrm{~s}^{-1}$ in $10 \mathrm{keV}$ tokamaks, the magnitude of the electric force remains typically negligible in front of that of the magnetic force. Electrons are dominantly governed by the magnetic force in tokamak plasmas and are guided by the magnetic field lines associated to the total magnetic field $\mathbf{B}=\mathbf{B}_{0}+\boldsymbol{\delta} \mathbf{B}$. This may no longer be true for ions, and even less for the most massive ions, that are comparatively more sensitive to the electric force, $q \mathbf{E}$, than to the force due to the magnetic perturbation, $q \mathbf{v} \times \delta \mathbf{B}$. However, as the electrons are the swiftest plasma particles, their confinement is essential. They play a leading role in the highest frequency phenomena of MCF. Because the electrons are dominantly controlled by the total magnetic force, $q \mathbf{v} \times \mathbf{B}$, they will be the most sensitive to, possibly transient, failures in the magnetic confinement, that is the backbone of MCF plasmas, with potentially deleterious consequences. This motivates a further, and ultimate, reduction of the physical picture of MCF plasmas: the purely magnetic approach.

Because $\mathbf{B}$ is divergence-free, it can be shown that, at each given time, the magnetic field lines are trajectories of a Hamiltonian system $H\left(x_{1}, x_{2}, x_{3}\right)$ depending on the space coordinates. Yet, chaos theory tells us that the minimal number of degrees of freedom required for the possible emergence of chaos is three. Therefore, in generic situations where no symmetry reduces the space dimension, magnetic field lines are expected to be spatially chaotic. The adverb spatially serves to remind that one refers here to the chaos of magnetic field lines at given times. One could also equivalently talk of Eulerian chaos [7]. We shall now address the questions of the existence and importance of spatially chaotic magnetic field lines in tokamak plasmas. In section 2, some numerical evidence for the existence of spatially chaotic magnetic field lines about the collapse phase of tokamak sawteeth with incomplete reconnection will be presented using the results of extensive test particle simulations. In tokamak sawteeth, the relative magnetic perturbation $\delta B / B$ may reach a few percents. This does not apply to tokamak operating regimes dominated by turbulence where $\delta B / B$ is not larger than $10^{-4}$. The final section 3 will however bring evidence for the existence of magnetic chaos in this case and briefly discuss some of its implication.

\section{Macroscopic features: indirect evidence for chaotic magnetic field lines in tokamak sawteeth with incomplete reconnection}

\subsection{The magnetic reconnection puzzle}

Magnetic reconnection may be defined as a sudden rearrangement of magnetic field that converts magnetic energy to 
plasma energy. Increasing observational evidence indicates that it is at play in the most spectacular space plasma phenomena, including solar flares [8], magnetospheric substorms [9] or gamma-ray bursts. A common denominator of their phenomenology is the sudden, explosive, nature of the reconnection onset. In this respect, there is an increasing interest in the role and impact of stochasticity, and possibly turbulence, in space magnetic reconnection [10]. Indeed, this could be an ingredient explaining the long-standing and controversial conundrum of the rapidity of the reconnection [11].

Magnetic reconnection does not show up only in space plasmas but has most important manifestations in toroidal magnetic confinement devices for fusion applications. It is there at play in sawteeth $[12,13]$, that were diagnosed for the first time in the early 1970s [14]. In this regime, quantities like the core plasma density or temperature undergo a slow rise before suddenly crashing on a periodic basis, so that the resulting time trace of these quantities resembles the edge of a saw. This relaxation phenomenon, that takes place in tokamaks, but also in reversed field pinches [15] or stellarators [16], is of considerable concern since the best fusion performances are attained just before the crashes. It puts therefore a limit to the fusion yield.

One possible explanation for the origin of sawtooth crashes has been the so-called stochastic scenario. Lichtenberg argued in a seminal paper [17] that the fast disruptive relaxation could be caused by the intrinsic large-scale stochasticity caused by overlapping magnetic islands. Despite some recent analogous stochastic interpretation of sawteeth experiments done in the ASDEX tokamak [18, 19], this theory faces a major, still unsolved, challenge due the puzzling outcome of a well-known experiment. In 1997, striking results were published [20] of some experiments performed in JET on the transport of nickel impurities during the sawtooth regime. Being introduced by the laser blow-off technique, trace nickel impurities were observed to propagate rapidly inwards until halting at a radius close to the $q=1$ surface, behaving then as a transport barrier, at which their density peaked. Then, coincident with the sawtooth collapse, nickel ions were suddenly transported towards the plasma core and, correspondingly, the soft x-ray emission indicated that the nickel density profile flattened during the very short, about $50 \mu \mathrm{s}$, lapse of time of the crash phase. The redistribution of these heavy impurities is as fast as the drop of the electron temperature occurring during the crash, even if electrons are two order of magnitude faster than $\mathrm{Ni}$ ions. This fact was given as an argument to rule out the stochastic nature of the transport during the sawtooth [20].

A well-known problem faced when studying the sawtooth phenomenon lies in the computational difficulty to reproduce it self-consistently. Magnetohydrodynamic-based approaches repeatedly predict a complete reconnection at each sawtooth cycle (in the absence of ad-hock tricks) whereas the experimental reconnection is incomplete. (See e.g. the presentation on sawtooth oscillations by Biskamp [21].) This may question the validity of fluid models in the reconnection collapse phase. Moreover, the treatment of the different species (electrons, plasma ions and impurities) in this kind of calculations is very heterogeneous. Specific ad hoc and phenomenological terms are added to the transport equations of each species, to approximate the expected behavior of the system. This approach tends to oversight the fact that at the very last, all particles in the plasma are subject to the same electric and magnetic fields, which will ultimately determine their dynamics. Another problem lies in the experimental difficulty to resolve the magnetic spectrum poloidally. Usually it is only toroidally resolved. All this led us to investigate sawtooth collapses in a new, indirect way, by making use of experimental results on the behavior of plasma and heavy trace particles as a testbed to probe the existence and the importance of the spatial chaos of magnetic field lines in sawtooth collapses.

In the framework of this study, presented below, nickel ions, electrons and plasma ions are considered as test particles, the trajectories of which will be computed using a full orbit following code. The only necessary information to perform this is having a prescribed model set for the electric and magnetic fields during the sawtooth collapse phase. The impact of spatial chaos will be eventually singled-out by considering sawtooth collapse models with integrable and spatially chaotic magnetic field lines for the same amplitude of the magnetic perturbation $\delta B$ in the same constant equilibrium magnetic field. Inward propagation of nickel ions during the collapse is recovered in the chaotic case, but not for integrable magnetic field lines.

\subsection{Framework}

We begin by noting that, due to the magnetic field being globally divergence-free, its field lines are the trajectories of a Hamiltonian system. Let us consider a set of variables $(\rho, \theta, \phi)$ where $\rho$ is a radius-like variable that vanishes along the magnetic axis. Then, there exist [22] two single-valued functions $\Psi(\rho, \theta, \phi)$ and $\Phi(\rho, \theta, \phi)$, such that the magnetic field can be written in the form

$$
\mathbf{B}=\nabla \Psi \times \nabla \theta+\nabla \phi \times \nabla \Phi .
$$

Eliminating the variable $\rho$ by using $\Phi(\Psi, \theta, \phi)$, the equations for the magnetic field lines read $\mathrm{d} \Psi / \mathrm{d} \phi=-\partial \Phi / \partial \theta$ and $\mathrm{d} \theta / \mathrm{d} \phi=\partial \Phi / \partial \Psi$, so that $\Phi$ plays the role of the Hamiltonian, the toroidal angle $\phi$ is a time-like variable and $\Psi$ is the momentum conjugated to the poloidal angle $\theta$. In a nonsteady state, the field-line Hamiltonian depends also on the real time $t$, that is a parameter not a canonical variable, and will be noted $\Phi^{t}$. As usual in tokamak physics, one will assume that the magnetic field $\mathbf{B}(\mathbf{r}, t)$ remains sufficiently close to some equilibrium $\mathbf{B}_{0}(\mathbf{r})$ with perfect magnetic surfaces. In Fourier decomposed form, the magnetic field-line Hamiltonian reads then

$$
\Phi^{t}(\Psi, \theta, \phi)=\Phi_{0}(\Psi)+\sum_{m, n} \Phi_{m n}^{t}(\Psi) \mathrm{e}^{\mathrm{i}(m \theta-n \phi)} .
$$

The magnetic field-line Hamiltonian associated to $\mathbf{B}_{0}$ is integrable, being $\Phi_{0}(\Psi)$. It identifies with the poloidal 

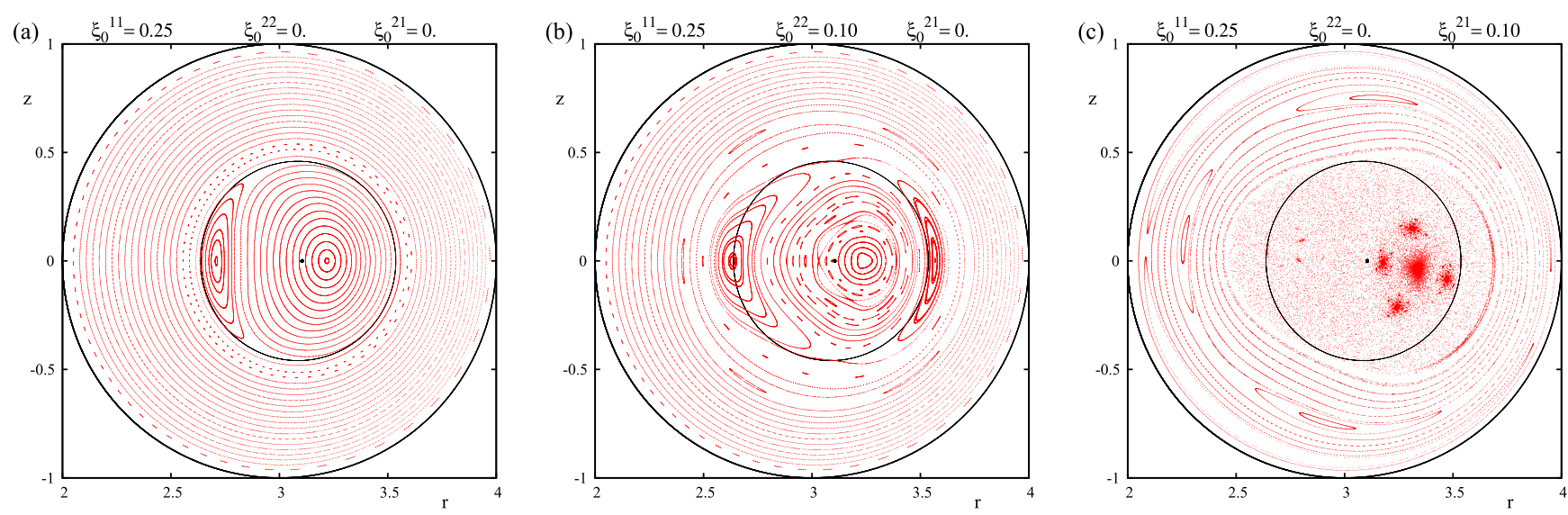

Figure 1. Comparison between the integrable and the chaotic cases: Poincare sections of the $\mathbf{B}$ lines at the crash onset in the case of integrable magnetic field lines with a $(1,1)$ displacement from the circular cross-section center of $25 \mathrm{~cm}(\mathrm{a})$, in the case of integrable magnetic field lines with a (1,1) displacement of $25 \mathrm{~cm}$ and a $(2,2)$ displacement of $10 \mathrm{~cm}$ (b) and in the case of locally chaotic magnetic field lines (c) with a (1,1) displacement of $25 \mathrm{~cm}$ and a $(2,1)$ displacement of $10 \mathrm{~cm}$. The last two cases have the same $\delta B / B$. The inner black circles and dots respectively mark the location of the $q=1$ surface and equilibrium magnetic axis.

magnetic flux and $\Psi$ with the toroidal magnetic flux. The safety profile $q(\Psi)$ is defined by $\mathrm{d} \Phi_{0} / \mathrm{d} \Psi=q(\Psi)^{-1}$.

Important to our study is to recognize that there exist also non-axisymmetric integrable field-line Hamiltonians obtained from equation (8). For instance, if one assumes that the magnetic field perturbation affects only $m=n$ modes, such as the dominant $(1,1)$ and $(2,2)$ modes, then the Hamiltonian may be expressed as a function of just two coordinates, namely $\Psi$ and the helical angle $\theta-\phi$, and is thus integrable [23, 24]. Then, in order to isolate the effect of the magnetic field line chaos on the motion of the nickel ions during the sawtooth crash, two different sets of magnetic field perturbations will be used [25]. In the first one, the perturbation has a single helicity with $(1,1)$ or $(1,1)+(2,2)$ modes associated to an integrable magnetic field-line Hamiltonian. In the second scenario, which is more realistic, the perturbation includes the $(1,1)$ modes and $m \neq n$ modes. The dominant $m \neq n$ mode is the $(2,1)$ mode that is linearly destabilized together with the $(1,1)$ mode due to toroidal effects.

It is likely that, in the JET discharges of [20], sawteeth were triggered by excitation of the internal kink mode in the semi-collisional ion-kinetic regime. However, as we focus on the crash phase, the duration of which is much shorter than that of the whole sawtooth cycle, and on the specific impact of the chaos of magnetic field lines, we are not interested here in the precise determination of the time development and space dependence of magnetic modes before the crash. Moreover, the crash phase being much shorter than the resistive time, the ideal Ohm's law will be considered as approximately valid. Denoting by $\boldsymbol{\xi}$ the plasma displacement field, the perturbed magnetic and electric field can be written as

$$
\begin{gathered}
\delta \mathbf{B}=\nabla \times\left(\boldsymbol{\xi} \times \mathbf{B}_{0}\right), \\
\mathbf{E}=-\frac{\partial \boldsymbol{\xi}}{\partial t} \times \mathbf{B} .
\end{gathered}
$$

So doing, the perturbed e.m. fields are derived under the sole knowledge of the plasma displacement field. The time evolution of the displacement is phenomenologically reconstructed as in [18, 26, 27]. In the JET discharges of [20], sawteeth are in all likelihood triggered by excitation of the $n=1$ internal kink mode, whereas higher modes are nonlinearly triggered $[24,28]$ as the $n=1$ mode develops. Higher order $m \neq n$ modes have even been experimentally measured [29]. The different nature of the magnetic field topology in both cases is illustrated by the Poincaré section plots for the integrable (mode $(1,1)$ ) and stochastic (modes $(1,1)$ and $(2,1))$ cases as shown in figure 1 . The radial displacement of the mode $(m, n)$ is written as

$$
\xi_{r}^{m n}(r, t)=\xi_{0}^{m n}(t) f^{m n}(r) \cos \left(m \theta-n \phi+\chi_{m n}(t)\right)
$$

and the other components of the displacement are obtained considering an incompressible displacement $\nabla \cdot \boldsymbol{\xi}=0$ and minimizing the change in potential energy for internal modes as in [27]. The effect of the crash is to quench the magnetic perturbation, although not completely, since, consistently with partial reconnection, a remnant of the $m=n=1$ island is commonly observed. Consequently, it will be assumed that, at the end of the crash phase, the mode amplitudes become $c_{m n} \xi_{0}^{m n}$, with a finite $(1,1)$ mode, given by $c_{11}=0.1$, while $c_{m n}=0$ for $(m, n) \neq(1,1)$. As for the radial dependence, the $f^{m n}(r)$ will be phenomenologically reconstructed as in $[18,26,27]$. Consistently with the experimental measurements obtained e.g. in ASDEX [19], the $(1,1)$ mode will have the form of the classical kink so that $f^{11}(r)$ amounts to a smoothed step function. As for the $(2,2)$ mode, that is nonlinearly triggered [24], an experimental reconstruction [18] will be used as in $[26,27]$. As for the other modes, the ideal linear cylindrical form will be used as in [30] so that

$$
f^{m n}(r)=\frac{r}{R_{0}}\left(\frac{r}{r_{s}}\right)^{m-1}\left(\frac{1}{q(r)}-\frac{n}{m}\right) \Theta\left(\frac{m}{n}-q(r)\right),
$$

where $q(r)$ is the equilibrium safety profile, chosen here as a 


\section{a) mode $(1,1)$ without $E$ field}

\section{b) mode $(1,1)$}

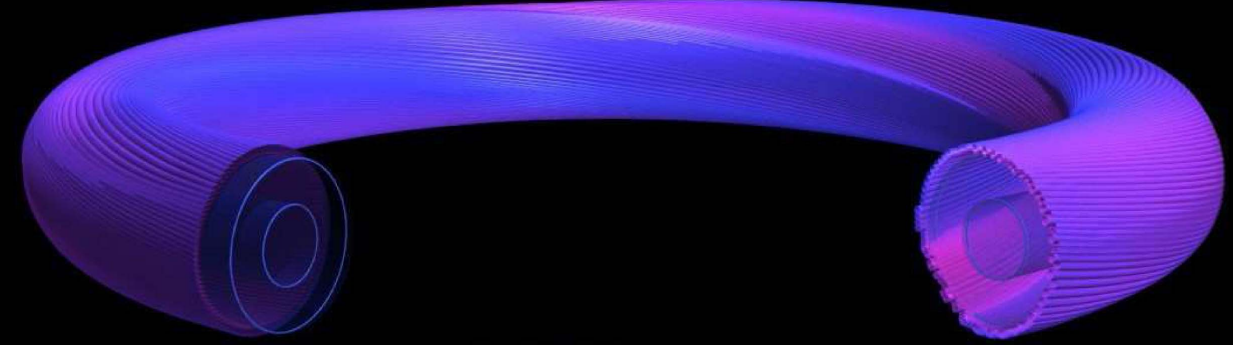

Radius (m)
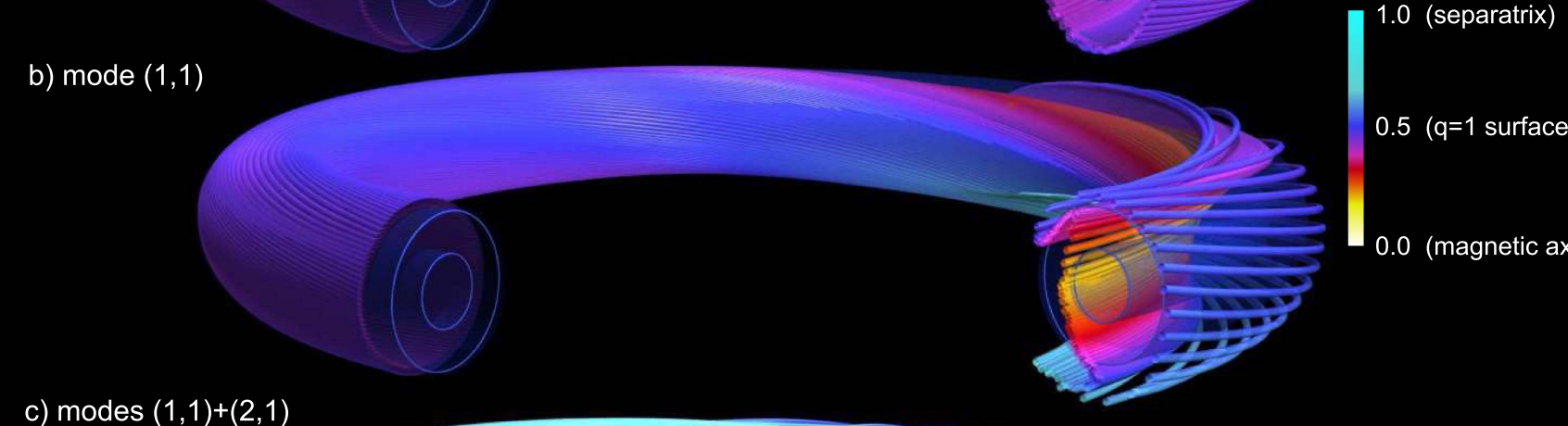

0.5 ( $q=1$ surface)

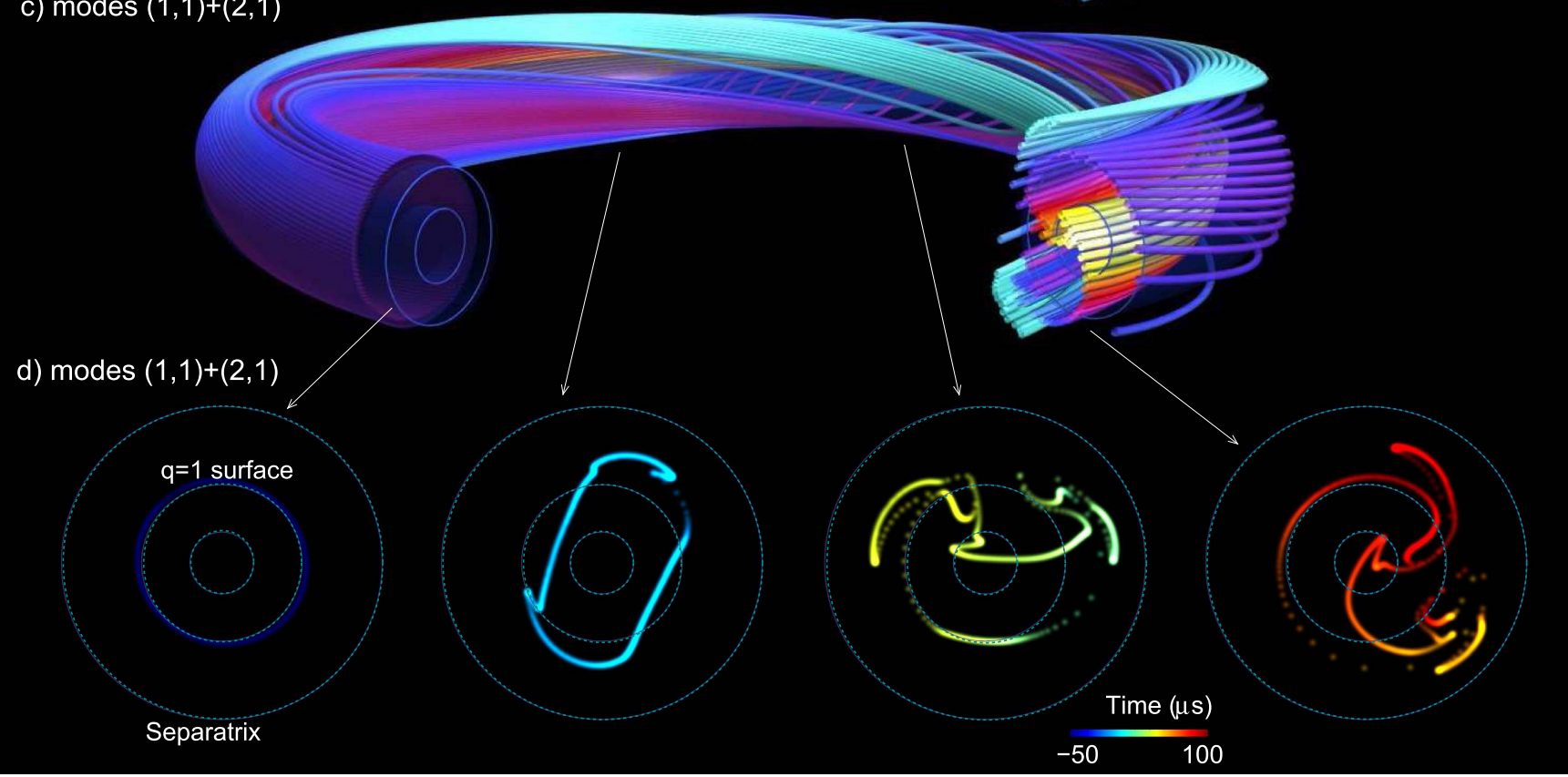

Figure 2. Trajectories of nickel ions during the sawtooth collapse. Trajectories between $t_{0}=-50 \mu \mathrm{s}$ and $t_{\mathrm{f}}=100 \mu \mathrm{s}$ are shown for the integrable case without (a) and with (b) electric field and for the chaotic case (c) with the electric field. (d) Cross sections $(R, z)$ taken from (c) showing integrated particle positions for the chaotic case for toroidal angles $\phi=0, \pi / 2, \pi$ and $3 \pi / 2$ are also shown in a time-dependent color scale. Nickel ions are injected at $\phi=0$, and with minor radius $r=0.5 \mathrm{~m}$.

monotonously increasing function with a central value equal to $q(0)=0.8$, where $r_{s}$ is defined by $q\left(r_{s}\right)=1$ and $\Theta$ denotes the Heaviside distribution. The canonical form of the perturbed Hamiltonian in equation (8) may be simply obtained by replacing $r$ by its approximate cylindrical form $r=a \sqrt{2 \Psi}$. Finally, the wave phases are modeled by $\chi_{m n}(t)=$ $\chi_{m n}^{0}-\omega_{m n} t$ with frequencies $\omega_{m n}$ taken in a realistic parameter window. The computations presented here use $\omega_{11}=5 \times 10^{4} \mathrm{rad} \mathrm{s}^{-1}$ and experimentally relevant ratios $\omega_{m n} / \omega_{11}$. JET-like parameters have been used: the minor radius is $a=1 \mathrm{~m}$, major radius is $3 \mathrm{~m}$ and the toroidal field $B_{\mathrm{T}}=2.8 \mathrm{~T}$.
Each nickel test particle obeys the equation of motion

$$
m \frac{\mathrm{d}^{2} \mathbf{r}}{\mathrm{d} t^{2}}=q \frac{\mathrm{d} \mathbf{r}}{\mathrm{d} t} \times \mathbf{B}+q \mathbf{E}
$$

where $\mathbf{B}=\mathbf{B}_{0}(\mathbf{r})+\delta \mathbf{B}(\mathbf{r}, t)$ and equations (9) and (10) are used as functions of the radial component of the plasma displacement with components (11). The charge number of the nickel ions has been retained to be $\mathcal{Z}=+26$, which appears to be the main ionization state present in similar experiments [31, 32] and their mass is $m=58.7 \mathrm{u}$. The full equation of motion (13) has been integrated without any gyroaveraging procedure for a collection of nickel ions using 


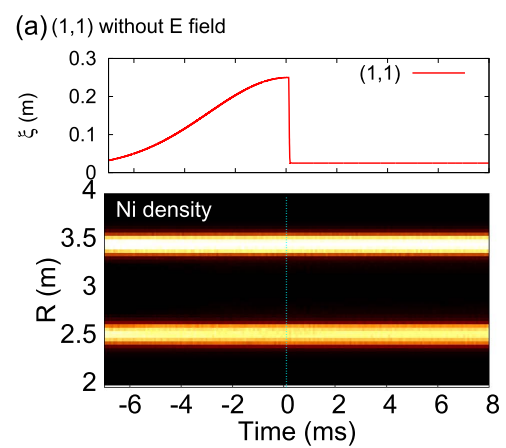

(b) $(1,1)$ with E field

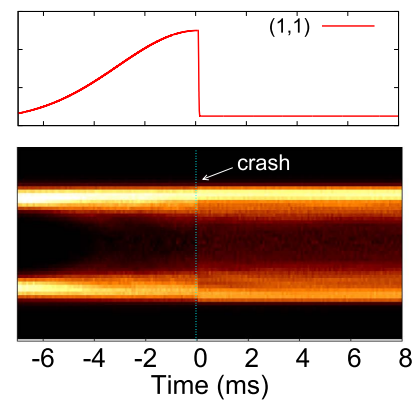

(c) $(1,1)+(2,2)$ with E field

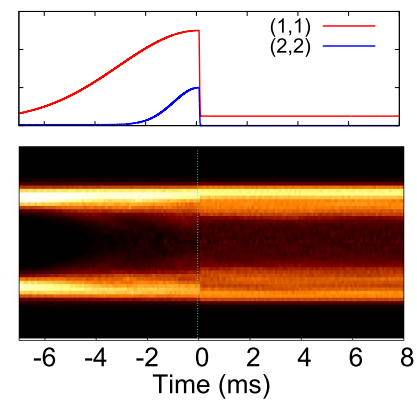

(d) $(1,1)+(2,1)$ with E field
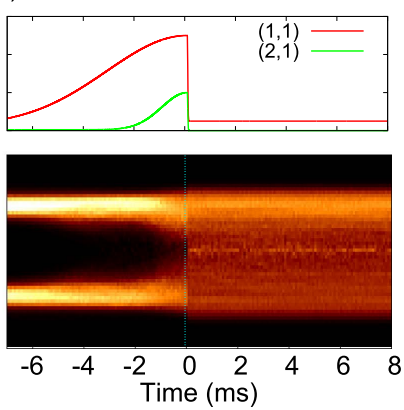

Figure 3. Evolution of Nickel ions during one sawtooth cycle. Top row: mode amplitude time dependence. Bottom row: ion density along the central horizontal chord evolution. Ions are initially uniformly distributed around the $q=1$ surface, specifically in a $0.5 \mathrm{~m}$ radius $-0.1 \mathrm{~m}$ thickness ring centered at the magnetic axis. The plots (a), (b) and (c) correspond to cases with integrable magnetic field lines. The right plot (d) corresponds to the chaotic magnetic field topology.

a fourth-order Runge-Kutta algorithm. Electrons trajectories are also integrated without gyroaveraging, but because of the very large number of time steps $\left(\sim 10^{9}\right)$ and to improve energy conservation, a Boris integrator was used instead of the Runge-Kutta integrator. The number of test particles in the calculation was $10^{7}$ for ions and $10^{5}$ for electrons. Initial velocity distribution for all particles in the calculations shown in the paper was isotropic with an energy of $8 \mathrm{keV}$. Calculations performed for lower energies $(2,4$ and $6 \mathrm{keV})$ show essentially the same trends than at $8 \mathrm{keV}$.

\subsection{Results on the nickel dynamics}

Typical orbits are represented in figure 2, for the integrable case (figures 2(a) and (b)) and the stochastic one (figure 2(c)). To reproduce the near-crash conditions of [20], $N$ nickel particles are uniformly placed at $t=0$ about the $q=1$ surface at distances of $0.5 \mathrm{~m}$ from the magnetic axis. The displacement used for the $(1,1)$ mode is $25 \mathrm{~cm}$, close to the value of the maximal displacement that may be inferred from the inspection of the tomographic reconstruction of central soft $\mathrm{x}$-ray emission on the figure 4 of [20]. The maximal amplitude of the perturbation $\delta B^{11} / B_{0}$ is $\sim 3.5 \%$. The crash starts at $t=0$ and finishes at $t=50 \mu \mathrm{s}$. As expected, if the $-\mathbf{v} \times \mathbf{B}$ electric field is neglected (figure 2(a)), the trajectories are not affected by the collapse of the sawtooth instability. The inclusion of the electric field in the integrable case clearly enhances the effect of the crash over the trajectories (2(b)). A fraction of the Ni ions are smoothly deflected towards the magnetic axis.

In the chaotic case including the electric field (2(c)), the crash produces a dramatic and complex redistribution of the particles. Some ions reach the magnetic axis whereas others are pushed away towards the separatrix. The trace of ions in four poloidal sections corresponding to the chaotic case (2 (d)) illustrates the sudden redistribution of ions. In less than $50 \mu \mathrm{s}$, the Ni ions experience a radial displacement as large as $0.5 \mathrm{~m}$. During the crash, the radial transport is driven by the large electric field (proportional to $\partial \boldsymbol{\xi} / \partial t$ ) associated with the rapid drop of the displacement. As the magnetic field is mainly toroidal and the displacement radial, the $\mathbf{E} \times \mathbf{B}$ drift is mainly in the radial direction. However, calculations show that the process is not uniquely determined by the $\mathbf{E} \times \mathbf{B}$ drift. For example, the trajectories do change when changing the charge sign or the velocity, both properties not affecting the $\mathbf{E} \times \mathbf{B}$ drift. Around the crash, the redistribution process can be characterized as superdiffusive, the mean square displacement being $\left\langle r^{2}\right\rangle \propto t^{\alpha}$ with $\alpha \sim 3>1$. Importantly, superdiffusive transport has been also reported in the context of astrophysical plasmas reconnection [33].

To get a more complete comparison between the prediction of our model and the experimental data we perform calculations for the whole sawtooth cycle. We take as initial condition ions distributed around the $q=1$ surface as found in [20]. Time evolution of the nickel density along the central horizontal chord $(z=0)$ for different scenarios is shown in figure 3 . When the mode $(1,1)$ is included and the electric field is neglected (3(a)), ions do not show a noticeably response to the perturbation of the magnetic field. The inclusion of the electric field (3(b)) enhances the radial diffusion of ions towards the axis in the growing phase of the instability. The electric field before this stage is mainly due to the temporal variation of the $\mathbf{B}$-field due to the mode rotation. The fastest the rotation, the stronger will be the diffusion of the impurities before the crash. The crash also contributes to the ion penetration, although the difference between the radial distribution before and after the crash is not marked.

The addition of the mode $(2,2)$ with a maximum displacement of $10 \mathrm{~cm}$ has little effect over the dynamics of the nickel impurities (3(c)). Note that the magnetic system including the modes $(1,1)$ and $(2,2)$ is still integrable. The inclusion of the mode $(2,1)$, i.e. the stochastic case, with the same maximum displacement, rotation frequency and growing time as the $(2,2)$ results in a strong inward diffusion of nickel impurities during the crash. In the cases of non-chaotic magnetic field lines, there is no noticeable nickel invasion towards the tokamak magnetic axis within the timescale of the sawtooth collapse. Conversely, quite significantly high fraction of nickel particles inside the core may be obtained when magnetic field lines display spatial chaos. Two features observed in experiments $[20,34]$ are retrieved in this calculations: (1) nickel ions do not penetrate into the tokamak core in the growing phase of the sawtooth, and (2) they suddenly diffuse to the magnetic axis during the collapse. The 


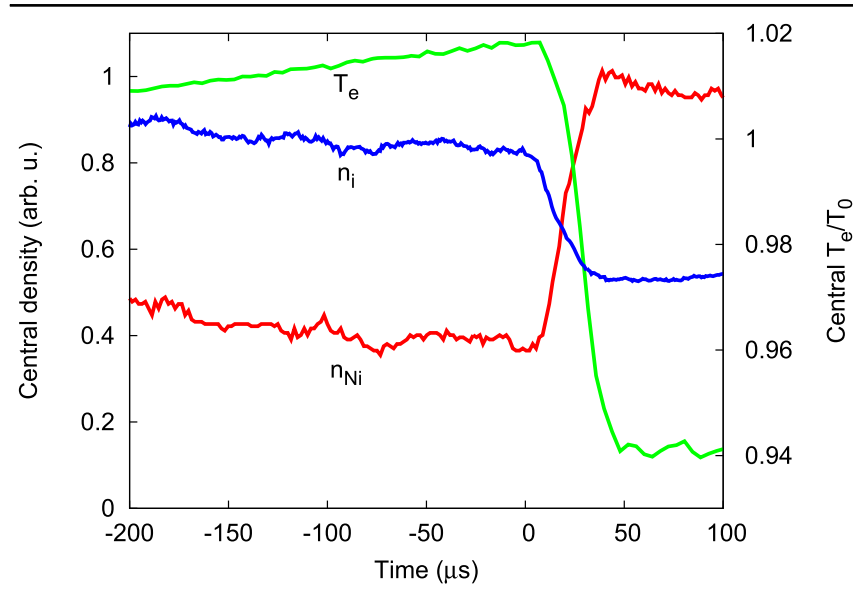

Figure 4. Temporal evolution of central electron temperature, central plasma ion density and central nickel density. The sawtooth collapse starts at $t=0 \mu \mathrm{s}$ and finishes at $t=50 \mu \mathrm{s}$.

combined result is the inversion of the sawtooth detected from soft x-ray emission, i.e. the signal grows instead of dropping at the crash.

To single-out the impact of magnetic chaos, we shall address the following question: for the same $|\delta \mathbf{B}|$, could we have obtained an inward nickel diffusion in a model with no chaos of the magnetic field lines? Calculations of the transport during the whole sawtooth cycle for a variety of parameters shows that nickel ions can reach the magnetic axis without the mode $(2,1)$ only for very large radial displacement of the $(1,1)$ mode $\left(\xi_{0}^{11}>35 \mathrm{~cm}\right)$, i.e. larger $|\delta \mathbf{B}|$, or for high rotation frequencies (tens of $\mathrm{kHz}$ ). But the behavior is very different than in the stochastic case. There is not a sudden diffusion of nickel during the collapse but a smooth diffusion during all the growing phase of the instability. Moreover, it is important to point out that, due to the toroidal magnetic field decreasing with the major radius as $1 / R$, there is some poloidal asymmetry in the transport. This is visible for nickel ions in figure 3(d). Consequently, the transport toward the magnetic axis observed with chaotic magnetic field lines is more effective from the direction of greater magnetic field.

\subsection{Consistency with experimental results}

Electron thermal velocity is more than 300 times larger than that of nickel ions, but the characteristic times for electron and impurity transport during the collapse found in experiments are similar. In other words, the collapse time for normal and inverse sawtooth inferred from SXR emission are comparable $[20,34]$. We also explored the transport of electrons and positive ions $\left(\mathrm{H}^{+}\right)$during the collapse, to test if a chaotic model for the magnetic field lines is compatible with a global dynamic independent of the particle velocity and mass. This will be presented elsewhere. Integrating the exact electron trajectories, we could reproduce electron temperature patterns very close to that found in electron temperature measurements by ECE $[35,36]$ using the e.m. with chaotic magnetic field lines for which the experimental nickel behavior was reproduced. The time evolution of the central temperature drop predicted by our model is close to that of the nickel penetration to the central region (see figure 4). Both times are close to the collapse time, $50 \mu \mathrm{s}$, in agreement with experimental evidence. Note that the temperature drop found $(\sim 8 \%)$ is weaker than in experiments $(\sim 30 \%)$, because the temperature profile created by the sawtooth during the time lapse integrated here is much flatter than the one produced by ohmic heating.

To summarize the outcome of our numerical simulations, we can say that in the case of integrable models of magnetic field lines for the sawtooth collapse and realistic parameters, nickel ions remained close to the regular magnetic surfaces, a feature clearly at odds with the experiments. Conversely, in the case of collapse models involving different helicities with mode amplitudes sufficiently large to make magnetic field lines stochastic in a region about the $q=1$ surface where nickel ions are concentrated before the crash, nickel ions did invade and remain in the core as observed in [20]. Moreover, simulations show that both the central electron temperature and plasma density evolve in the same timescale.

Eventually, the puzzle pointed by Wesson [20] on the nickel and electron populations moving radially on the same timescale appears to have a simple explanation: do to their mass difference, while electrons dominantly feel (and act back on) the magnetic force being redistributed spatially by the chaos of magnetic field lines at the collapse, the motion of nickel ions is more affected by the electric force and mostly follows the $\mathbf{E} \times \mathbf{B}_{0}$ drift. Due to Faraday's law, the magnetic perturbation and electric field act on the same timescale, controlled by the swiftest plasma particles, namely by the electrons.

\subsection{Conclusion and future prospects}

This study offers a quantitative proof of principle that magnetic field lines are chaotic near the onset of sawtooth collapses, at least in the case of incomplete reconnection, that corresponds to spontaneous magnetic reconnection in the space plasmas terminology.

In this study, as in the original experimental work by Wesson et al [20], nickel ions take the role of, externally introduced, massive test particle impurities that serve to probe the physical mechanisms governing tokamak sawteeth. Nowadays, the fate of impurities in magnetic fusion devices has become an essential issue in itself. ITER will be equipped with tungsten plated divertors. Those plasma-facing components could be partially ablated under intense heat flux. In this respect, this work should, for instance, be useful in the modeling of the transport of tungsten impurities in ITER's sawteeth.

With respect to the general problem of magnetic reconnection, this work points to the existence of chaotic magnetic field lines in the last stage of magnetic reconnection. Although this evidence is given here in the tokamak setting, there is no obstacle for its occurrence in space configuration. Further exploration should be conducted to assess whether this spatial chaos of magnetic field lines may be the/one explanation to the rapidity of spontaneous magnetic reconnection. This chaos emanates here from a small number and not, as in turbulence, from a large spectrum of modes. As 


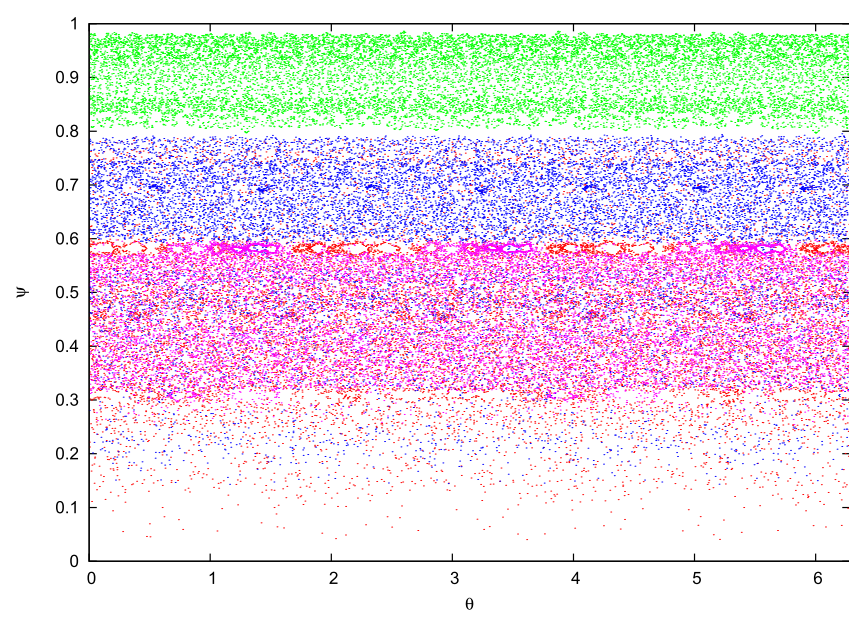

Figure 5. Puncture plots of four (colored online) magnetic field lines crossing some given poloidal tokamak cross-section for a magnetic spectrum with $\delta B / B \sim 3 \times 10^{-5}$. See [42] for details on the model. The representation is in the $(\theta, \psi)$ plane. One can observe some cantori about $\psi \simeq 0.7$ and $\psi \simeq 0.6$.

spontaneous magnetic reconnection may start from an instability, such a stage of low-dimensional chaos should precede turbulence and its impact on reconnection in the astrophysical context remains to be explored. A preliminary analysis indicates that, all other things being equal, chaotic magnetic field lines induce an increased rate of energy transfer compared with integrable (laminar) B lines [7].

\section{Microscopic features: microtearing turbulence}

We shall now consider the case where the relative magnitude of the magnetic field perturbation $\delta B / B$ is small but sustained by a wide spectrum of modes. This situation occurs in conditions of turbulence, according to the MCF terminology. In this case, magnetic field lines behave in a stochastic way, as many resonance modes overlap, in the usual tokamak operation conditions where $\delta B / B$ is of the order $10^{-4}[37,38]$. This was first inferred in the seminal work by Stix [39], reported in some recent gyrokinetic simulations [40, 41], and studied within some realistic microtearing magnetic model [42].

Figure 5 shows a Poincaré plot of magnetic field lines obtained with a monotonic $q$-profile and a full spectrum of tearing modes as in [42] with a magnitude of $\delta B / B$ as low as $3 \times 10^{-5}$. This clearly indicates that magnetic surfaces are destroyed, yet some transport barriers (KAM tori) and their remnants (cantori) located about low rational values of $q$ contain the radial diffusion of magnetic field lines.

For the values of $\delta B / B$ experimentally measured in Tore Supra [37] and recently in JIPPT-IIU [38], the flux across the cantori is so large that they effectively provide no barriers to transport and magnetic field lines tend to behave almost stochastically exploring all the phase space [42]. This intrinsic stochasticity of magnetic field lines justifies the validity of a Fokker-Planck-like approach to the electron transport, first proposed by Rechester and Rosenbluth [43]. This yields the estimate of the electron thermal diffusivity as $\chi_{e} \sim v_{\text {the }} D_{B}$, with $v_{\text {the }}$ the electron thermal velocity and $D_{B}$ the diffusion coefficient of the magnetic field lines. Let us note here that all the magnetic content is needed to estimate the transport, not only the small wavelength modes, 'turbulent part', that is sometimes only retained.

The point that we wish to make here is that the stochasticity of magnetic lines is not necessarily catastrophic for MCF plasma confinement: it is all a matter of confinement time, or equivalently here, a matter of magnitude of the magnetic diffusivity. The study [42] pointed out the sensitivity of the magnetic confinement time $\tau_{B}$ with $\delta B / B$ as $\tau_{B} \sim(\delta B / B)^{-2}$. Containing $\delta B / B$ to values low enough so that partial magnetic transport barriers still exist (as in figure 5) would lead to much higher electron confinement times, since, as discussed in the introduction, the electron transport identifies dominantly to the magnetic transport. This is presumably a path to improved fusion performances if this is performed jointly with the control of the electrostatic turbulence governing the ion transport.

\section{Acknowledgments}

This work was carried out within the framework of the French Research Federation for Fusion Studies. Some financial support from the ECOS-MINCyT Research Grant No. A09E02 is gratefully acknowledged.

\section{References}

[1] Galeev A A and Sagdeev R Z 1968 Transport phenomena in a collisionless plasma in a toroidal magnetic system JETP 26233

[2] Spitzer L 1956 Physics of Fully Ionized Gases (New York: Interscience)

[3] Firpo M-C Contributions to some hot plasma physics issues Habilitation à Diriger des Recherches Université Paris 6

[4] Mynick H E 2006 Transport optimization in stellarators Phys. Plasmas 13058102

[5] Mynick H E, Pomphrey N and Xanthopoulos P 2010 Optimizing stellarators for turbulent transport Phys. Rev. Lett. 105095004

[6] Bickerton R J 1997 Magnetic turbulence and the transport of energy and particles in tokamaks Plasma Phys. Control. Fusion 39339

[7] Firpo M-C, Ettoumi W, Lifschitz A F, Retinò A, Farengo R F, Ferrari H E and García-Martínez P L 2016 Impact of the Eulerian chaos of magnetic field lines in magnetic reconnection Phys. Plasmas 23122905

[8] Su Y, Veronig A M, Holman G D, Dennis B R, Wang T, Temmer M and Gan W 2013 Imaging coronal magnetic-field reconnection in a solar flare Nat. Phys. 9 489-93

[9] Angelopoulos V et al 2008 Tail reconnection triggering substorm onset Science 321 931-5

[10] Lazarian A, Eyink G, Vishniac E and Kowal G 2015 Turbulent reconnection and its implications Phil. Trans. R. Soc. A 3732041

[11] von Steiger R 2013 Space physics - grand challenges for the 21st century Frontiers Phys. 16 
[12] Zweibel E G and Yamada M 2009 Magnetic reconnection in astrophysical and laboratory plasmas Annu. Rev. Astron. Astrophys. 47 291-332

[13] Hastie R J 1997 Sawtooth instability in tokamak plasmas Astrophys. Space Sci. 256 177-204

[14] von Goeler S, Stodiek W and Sauthoff N 1974 Studies of internal disruptions and $m=1$ oscillations in tokamak discharges with soft-x-ray tecniques Phys. Rev. Lett. 33 1201-3

[15] Bonfiglio D, Veranda M, Cappello S, Escande D F and Chacón L 2013 Experimental-like helical self-organization in reversed-field pinch modeling Phys. Rev. Lett. 111 085002

[16] Cattanei G et al 1977 On sawtooth oscillations and MHD modes in the W VII A stellarator 8th European Conf. on Controlled Fusion and Plasma Physics (Prague, Czechoslovakia, 19-23 September) vol 1 p 127

[17] Lichtenberg A J 1984 Stochasticity as the mechanism for the disruptive phase of the $m=1$ tokamak oscillations $\mathrm{Nucl}$. Fusion 241277

[18] Igochine V, Dumbrajs O, Zohm H and the ASDEX Upgrade Team 2008 Transition from quasiperiodicity to chaos just before sawtooth crash in the ASDEX Upgrade tokamak Nucl. Fusion 48062001

[19] Igochine V, Boom J, Classen I, Dumbrajs O, Guenter S, Lackner K, Pereverzev G and Zohm H 2010 Structure and dynamics of sawteeth crashes in ASDEX Upgrade Phys. Plasmas 17122506

[20] Wesson J A, Alper B, Edwards A W and Gill R D 1997 Transport in the sawtooth collapse Phys. Rev. Lett. 79 5018-21

[21] Biskamp D 1997 Nonlinear Magnetohydrodynamics (Cambridge Monographs on Plasma Physics) (Cambridge: Cambridge University Press) p 260

[22] D'haeseleer W D, Hitchon W N G, Callen J D and Shohet J L 1991 Flux Coordinates and Magnetic Field Structure: A Guide to a Fundamental Tool of the Plasma Theory (Springer Series in Computational Physics) ed R Glowinski et al (Berlin: Springer)

[23] Firpo M-C and Constantinescu D 2011 Study of the interplay between magnetic shear and resonances using Hamiltonian models for the magnetic field lines Phys. Plasmas 18 032506

[24] Firpo M-C, Ettoumi W, Farengo R, Ferrari H E, García-Martínez P L and Lifschitz A F 2013 Development of magnetohydrodynamic modes during sawteeth in tokamak plasmas Phys. Plasmas 20072305

[25] Ettoumi W 2013 Dynamique hamiltonienne et phénomènes de relaxation: d'un modèle champ moyen au confinement magnétique $P h D$ Thesis Ecole Polytechnique

[26] Farengo R, Ferrari H E, Firpo M-C, García-Martínez P L and Lifschitz A F 2012 Alpha particle redistribution due to experimentally reconstructed internal kink modes Plasma Phys. Control. Fusion 54025007

[27] Farengo R, Ferrari H E, García-Martínez P L, Firpo M-C, Ettoumi W and Lifschitz A F 2013 Redistribution of high energy alpha particles due to sawteeth with partial reconnection Nucl. Fusion 53043012
[28] Firpo M-C and Coppi B 2003 Dynamical analysis of the nonlinear growth of the $m=n=1$ resistive internal mode Phys. Rev. Lett. 90095003

[29] Munsat T, Park H K, Classen I G J, Domier C W, Donné A J H, Luhmann N C Jr, Mazzucato E, van de Pol M J and The TEXTOR team 2007 Localization of the magnetic reconnection zone during sawtooth crashes in tokamak plasmas Nucl. Fusion $47 \mathrm{~L} 31$

[30] Zhao Y and White R B 1997 Simulation of $\alpha$-particle redistribution due to sawteeth on the tokamak fusion test reactor Phys. Plasmas 4 1103-9

[31] Pasini D, Giannella R, Taroni L L, Mattioli M, Denne-Hinnov B, Hawkes N, Magyar G and Weisen H 1992 Measurements of impurity transport in JET Plasma Phys. Control. Fusion 34677

[32] Giannella R, Lauro-Taroni L, Mattioli M, Alper B, Denne-Hinnov B, Magyar G, O'Rourke J and Pasini D 1994 Role of current profile in impurity transport in JET L mode discharges Nucl. Fusion 341185

[33] Lazarian A and Yan H 2014 Superdiffusion of cosmic rays: Implications for cosmic ray acceleration Astrophys. $J$. 78438

[34] Wei D, Yi L, Zhengying C, Yunbo D, Yuan H, Peng Z, Ping S and Bingzhong F 2007 Sawtooth activities during impurity injection by laser blow-off in HL-2A Plasma Sci. Technol. 9411

[35] Yamada M, Levinton F M, Pomphrey N, Budny R, Manickam J and Nagayama Y 1994 Investigation of magnetic reconnection during a sawtooth crash in a high-temperature tokamak plasma Phys. Plasmas 1 3269-76

[36] Liu W-D, Xie J-L, Azam H, Gao B-X and the EAST Team 2015 Electron cyclotron emission imaging observations of $m / n=1 / 1$ and higher harmonic modes during sawtooth oscillation in ICRF heating plasma on EAST Chin. Phys. Lett. 32065201

[37] Zou X L, Colas L, Paume M, Chareau J M, Laurent L, Devynck P and Gresillon D 1995 Internal magnetic turbulence measurement in plasma by cross polarization scattering Phys. Rev. Lett. 75 1090-3

[38] Hamada Y, Watari T, Nishizawa A, Yamagishi O, Narihara K, Ida K, Kawasumi Y, Ido T, Kojima M, Toi K and The JIPPT-IIU Group 2015 Microtearing mode (MTM) turbulence in JIPPT-IIU tokamak plasmas Nucl. Fusion $\mathbf{5 5}$ 043008

[39] Stix T H 1973 Magnetic braiding in a toroidal plasma Phys. Rev. Lett. 30 833-5

[40] Doerk H, Jenko F, Pueschel M J and Hatch D R 2011 Gyrokinetic microtearing turbulence Phys. Rev. Lett. 106155003

[41] Hatch D R, Pueschel M J, Jenko F, Nevins W M, Terry P W and Doerk H 2012 Origin of magnetic stochasticity and transport in plasma microturbulence Phys. Rev. Lett. 108235002

[42] Firpo M-C 2015 Microtearing turbulence: magnetic braiding and disruption limit Phys. Plasmas 22122511

[43] Rechester A B and Rosenbluth M N 1978 Electron heat transport in a tokamak with destroyed magnetic surfaces Phys. Rev. Lett. 40 38-41 